\title{
GASTROINTESTINAL BLEED
}

Gastrointestinal (GI) bleed refers to any bleeding that originates in the GI tract. Bleeding is generally defined as upper (between the mouth and ligament of Treitz) or lower (from the ligament of Treitz to the anus). Healthcare Cost and Utilization Project (HCUP) 2002 data for the Diagnosis Related Group (DRG) for GI Bleed with complications or co-morbidities reveals approximately 409,000 discharges with an in-hospital mortality of 3.0\%. The mean length-of-stay for these patients was 4.4 days, with mean charges of $\$ 15,000$. Hospitalists provide immediate care for these patients, who often require coordination of care across multiple specialties. Hospitalists lead quality improvement initiatives that optimize the efficiency and quality of care for these patients.

\section{KNOWLEDGE}

Hospitalists should be able to:

- Explain the multiple potential etiologies or pathophysiologic processes that lead to GI bleeds.

- Describe and differentiate the clinical features and presentations of upper and lower GI bleeds.

- Explain the differential diagnosis for the most common causes of upper and lower GI bleeds.

- Describe the indicated tests required to evaluate GI bleeds.

- Explain the risk factors for upper and lower GI bleeds, and clinical indicators of patients at high risk for complications.

- Explain the factors that may require early aggressive interventions or increase patient risk for recurrent bleeds.

- Risk stratify patients with GI bleeds and determine the level of care required.

- Describe the indications for transfusion therapy in GI bleeds, and explain the various methods of treatment for coagulopathy.

- Compare the advantages and disadvantages of medical, endoscopic, and surgical treatments for patients with upper and lower GI bleeds.

- Explain indications, contraindications and mechanisms of action of pharmacologic agents used to treat GI bleeds.

- Explain patient characteristics that on admission portend poor prognosis.

- Identify clinical, laboratory and imaging studies that indicate severity of disease.

- Explain goals for hospital discharge, including specific measures of clinical stability for safe care transition.

\section{SKILLS}

Hospitalists should be able to:

- Elicit a thorough and relevant history, including a directed medication, family and social history.

- Perform a physical examination and identify clinical indicators of upper and lower GI bleeds, and evidence of underlying states, which may include liver disease.

- Recognize physical findings that indicate clinical instability due to acute blood loss, including digital rectal examination, and interpretation of orthostatic blood pressure and pulse measurements.

- Insert a nasogastric tube, perform a gastric lavage, and interpret the results.

- Order and interpret results of appropriate laboratory, imaging, and endoscopic testing.

- Synthesize results of physical examination, laboratory and imaging studies to determine the best management and care plan for the patient.

- Formulate an evidence based treatment plan including nutritional recommendations, pharmacologic agents and dosing, and coordination of endoscopic and surgical interventions tailored to the individual patient.

- Determine frequency for laboratory monitoring and transfusion during hospitalization.

- Assure adequate intravenous access to allow rapid volume and blood product resuscitation.

- Perform rapid hemodynamic resuscitation.

- Recognize and treat signs of clinical decompensation and recurrent bleeding.

- Assess patients with suspected GI bleeds in a timely manner, and manage or co-manage the patient with the primary requesting service. 


\section{ATTITUDES}

Hospitalists should be able to:

- Communicate with patients and families to explain the disease etiology, prognosis, risk reduction strategies, and symptoms of recurrent GI bleed.

- Communicate with patients and families to explain goals of care plan, discharge instructions and management after release from hospital.

- Communicate with patients and families to explain risks, benefits, and alternatives to transfusion therapy.

- Communicate with patients and families to explain tests and procedures and their indications, and to obtain informed consent.

- Recognize the indications for early specialty consultation, which may include interventional radiology, gastroenterology and surgery.

- Initiate prevention measures including avoidance of NSAIDs, stress ulcer prophylaxis in critically ill patients, dietary modification, and evidence based medical therapies.

- Employ a multidisciplinary approach, which may include nursing, pharmacy and nutrition services, and specialty and referring physicians, to the care of patients with GI bleeds.

- Employ a multidisciplinary approach to the care of patients with GI bleed that begins at admission and continues through all care transitions.

- Establish and maintain an open dialogue with patients and families regarding care goals and limitations, including palliative care and end-of-life wishes.

- Address resuscitation status early during hospital stay; discuss and implement end-of-life decisions by patient or family when indicated or desired.

- Inform receiving physician of pending study results.

- Employ multidisciplinary teams to facilitate discharge planning and communicate to outpatient providers the notable events of the hospitalization and anticipated post-discharge needs.

\section{SYSTEM ORGANIZATION AND IMPROVEMENT}

To improve efficiency and quality within their organizations, Hospitalists should:

- Lead, coordinate or participate in the development and promotion of guidelines and/or pathways that facilitate efficient and timely evaluation and treatment of patients with GI bleeds.

- Lead, coordinate or participate in multidisciplinary teams, which may include emergency medicine physicians, gastroenterologists and nurses, to develop quality improvement initiatives that promote early identification of GI bleeds and reduce preventable complications.

- Develop systems that provide timely reports of pending study results to outpatient providers.

- Integrate outcomes research, institution-specific laboratory policies, and hospital formulary to create indicated and cost-effective diagnostic and management strategies for patients with GI bleeds. 